East African Medical Journal Vol. 87 No. 5 May 2010

SYPHILLIS AMONG PREGNANT WOMEN IN JUBA, SOUTHERN SUDAN 2009

S. K. Emmanuel, BSc, Senior Inspector, Public Health Laboratory, Ministry of Health of the Government of SouthernSudan, M. Lado, MBBS, MSc, Director of Tropical Endemic Diseases, Ministry of Health of the Government of Southern Sudan, S. Amwayi, MBChB, MSc, Epidemiology Track Field Coordinator FELTP-Kenya, A. M. Abade, BSc, MSc, Laboratory Track Field Coordinator FELTP-Kenya, J. O. Oundo, PhD, Laboratory Residents Advisor FELTP-Kenya/Deputy Laboratory Director (GDDD / IEIP Laboratory), CDC Kenya, P.O. Box 6200- 00200, Village Market, Nairobi, Kenya and J. R. Ongus, PhD, Lecturer, Medical Laboratory Sciences Department, Jomo Kenyatta University of Agriculture and Technology, P.O. Box 62000 - 00200, Nairobi, Kenya

Request for reprints to: Dr. J. O. Oundo, Deputy Laboratory Director (GDDD/IEIP Laboratory), CDC Kenya, P.O. Box 606-00621, Nairobi, Kenya

\title{
SYPHILIS AMONG PREGNANT WOMEN IN JUBA, SOUTHERN SUDAN
}

\author{
S. K. EMMANUEL, M. LADO, S. AMWAYI, A.M. ABADE, J.O. OUNDO and J.R. ONGUS
}

\begin{abstract}
Objective: Determine factors associated with syphilis among pregnant women.

Design: Cross-sectional study.

Setting: Antenatal clinics of Juba Teaching Hospital, Malakia National Health Insurance

Centre and Munuki Primary Health Care Centre in Juba, Southern Sudan.

Subjects: Consenting pregnant women not on syphilis treatment.

Main outcome measures: Socio-demographic and clinical data, knowledge and behavioural characteristics.

Results: Of the 231 pregnant women participants, $51(22.1 \%)$ were positive for syphilis with the rapid plasma reagin test and $79(34.2 \%)$ were positive with the treponema pallidum Haemagglutination assay. Risk factors for syphilis were: housewife (OR 2.808; $\mathrm{P}=0.0116$ ), abortion (OR 2.654; $\mathrm{P}=0.0116$ ) and partner travel ( $O R 2.149 ; \mathrm{P}=0.028)$. Attending antenatal clinic for previous pregnancy was protective $(O R=.281 ; P=0.0004)$ for syphilis.

Conclusions: This is the first study to determine the prevalence and associated factors in the three clinics in Juba, South Sudan. There is a high prevalence of syphilis in pregnant women attending the selected health facilities. Treponema Pallindum Haemagglulination Assay can be used as a field test for syphilis due to its high sensitivity and specificity. Health education, screening and treating positive expectant mothers can reduce the prevalence of syphilis.
\end{abstract}

\section{INTRODUCTION}

Syphilis is a chronic infectious disease caused by the spirochaete Treponema pallidum subspecies pallidum. It is a complex systemic disease with protean manifestations and virtually any organ in the body can be involved. Syphilis in a pregnant woman results in miscarriage, stillbirth, or a congenitally infected baby (1). The risk of vertical transmission reduces as maternal syphilis advances to late stages, but in early latent stage which is mostly asymptomatic the risk of vertical transmission remains about $30 \%$ to $60 \%$ (2). Serological screening and treating mothers for syphilis during pregnancy can prevent adverse pregnancy outcomes and vertical transmission associated with maternal infection (3). In 2004 World Health Organisation (WHO) estimates were that approximately 12 million new syphilis infections occur each year globally, many of which go untreated especially in less developed countries.

Syphilis in pregnancy is still a problem in some less developed countries as opposed to developed countries like United States and countries in Europe. Prevalence of syphilis around sub-Saharan Africa ranges between 2.5\% in Burkina Faso, $8.4 \%$ in South Africa, and $17.4 \%$ in Cameroon (4) and a high prevalence of $42 \%$ in Mozambique (5). In 1997studies amongst pregnant women reported by WHO in the North and North Eastern regions of Africa showed syphilis infection rates of $3.1 \%$ in Djibouti, $3 \%$ in Morocco and 2.4\% in Sudan.

The civil war that lasted for over two decades in Southern Sudan ended with devastating outcome of massive displacement, depletion of assets, and no or limited accessible established social services system and arrested development in a country which was 
already one of the most underdeveloped in the world. The health system in South Sudan is relatively new with the routine ANC screening programmes yet to be established. TheSouthernSudan household survey conducted in 2006 found that maternal and child mortality rates are among the world's highest.

The quality of Maternal and Child Health $(\mathrm{MCH})$ care is also very low. After the signing of the Comprehensive Peace Agreement (CPA) in Sudan, sentinel surveillance was carried out by Centers for Disease Control and Prevention in nine sites in Southern Sudan which revealed the prevalence of syphilis to range from $12 \%$ to $21 \%$ in pregnant women. There is, however, no data on the burden of syphilis among pregnant women in Juba as it was not included in the sentinel surveillance. The current study, therefore, intended to determine the prevalence of syphilis and associated factors in pregnant women attending selected antenatal clinics in Juba southern Sudan.

\section{MATERIALS AND METHODS}

This was a cross sectional study that was carried out in Juba southern Sudan between $1^{\text {st }}$ August to $30^{\text {th }}$ November, 2009. Juba is the capital city of Southern Sudan and is situated in Central Equatoria state which has a population of 1,118,233 people. This study was conducted in the antenatal clinics of Juba Teaching Hospital (JTH), Malakia National Health Insurance Centre (MNHIC), and Munuki Primary Health Care Centre (MPHCC). These clinics serve almost $70 \%$ of pregnant women visiting antenatal clinics in Juba, southern Sudan. Juba is divided into three main payams (sub counties) Juba, Kator and Munuki. The antenatal clinics are situated in the three sub-counties.

Using the formula $\mathrm{N}=\mathrm{Z}^{2} \mathrm{pq} / \mathrm{d}^{2}$ for estimation of population proportions, the minimum sample size was calculated to be 226 study participants.

Consenting pregnant women not on syphilis treatmentattending antenatal clinics of JTH, MNHIC and MPHCC were considered eligible for this study. Data on socio-demographic characteristics, clinical history, behavioural characteristics and knowledge was collected using a semi-structured questionaire. Five millilitres of blood was drawn from each participant and from which plasma was obtained. The specimens were screened using Rapid Plasma Reagin (RPR) kit (Fortress Diagnostic Ltd, UK) to detect the non-specific antibodies in the blood of the patient that may indicate that T. pallidum is present.
This test does not look for antibodies against $\mathrm{T}$. pallidum, but rather for antibodies against substances released by cells damaged by T. pallidum. A positive test was seen as flocculation within eight minutes. The addition of carbon particles which take no part in the reaction assists in the visualization of the flocculation and a negative test was seen by the absence of the flocculation. Treponema Pallidum haemagglutination Assay (TPHA) (Randox Ltd. Antrim, UK) was used as a confirmatory test. TPHA is an indirect Haemagglutination assay used to detect specific antibodies against T. pallidum. The reagent system consists of avian red blood cells coated with antigen of T. pallidum. Avian cells are used as they are nucleated and therefore heavier, producing a faster reaction time.

Data were entered and analysed using Epi Info ${ }^{\mathrm{TM}}$ Version 5 statistical software (CDC Atlanta, USA). Frequency distributions were performed on sociodemographic variables and RPR status of the study population. TPHA status was then cross tabulated with socio-demographic variables. Odds ratios with 95\% confidence intervals and chi square test were used to assess association of variables with syphilis status. An odds ratio of $<1$ was taken to be protective while $>1$ was taken as a risk factor. An odds ratio of " 1 " indicated that there was no difference between the study groups with the outcome variable under study. A p-value $\leq 0.05$ was considered to support statistically significant association.

\section{RESULTS}

In this study, a total of 231 consenting study participants comprising of $91(40 \%)$ from Juba Teaching Hospital, 80 (35\%) from Malakia National Health Insurance Centre and 60 (25\%) from Munuki Primary Health Care Centre respectively were recruited into the study on a proportionate basis dependent on the population of pregnant women visiting their antenatal clinics. The participants ages ranged from 15 to 37 years (mean age $24 \pm 4.8$ years, median age $=23$ years) and a gestation period ranging from six to thirty seven weeks ( $24 \pm 7.5$ weeks). Those who attained primary level education were $34.6 \%$ and those with no education were $30.7 \%$ of the participants.

A majority $(76.5 \%)$ of the participants were housewives and the participants were married monogamous $(63.6 \%)$ or polygamous $(30.3 \%)$ or single $(4.3 \%)$. Details of the socio- demographic data are outlined in Table 1. 
Table 1

Socio-demographic characteristics of study participants

\begin{tabular}{|c|c|c|c|c|c|}
\hline Variable & $\begin{array}{l}\mathrm{JTH} \\
\mathrm{n}=(91)\end{array}$ & $\begin{array}{l}\text { MNHIC } \\
\mathrm{n}=(80)\end{array}$ & $\begin{array}{l}\text { MPHCC } \\
\mathrm{n}=(60)\end{array}$ & \multicolumn{2}{|c|}{$\begin{array}{l}\text { Total } \\
\text { No. }(\%)\end{array}$} \\
\hline Age range & $15-37$ & $15-37$ & $15-37$ & & \\
\hline Mean age & 24 & 24 & 24 & & \\
\hline Std Deviation & \pm 4.7 & \pm 5.0 & \pm 5.0 & & \\
\hline Median & 24 & 23.5 & 24 & & \\
\hline \multicolumn{6}{|l|}{ Educational level } \\
\hline None & 25 & 24 & 22 & 71 & 30.7 \\
\hline Primary & 34 & 29 & 17 & 80 & 34.6 \\
\hline Secondary & 26 & 18 & 13 & 57 & 24.7 \\
\hline Tertiary & 6 & 9 & 8 & 23 & 10.0 \\
\hline \multicolumn{6}{|l|}{ Occupation } \\
\hline Employed & 16 & 10 & 9 & 35 & 15.2 \\
\hline Housewife/Unemployed & 66 & 64 & 46 & 176 & 76.5 \\
\hline Informal employment & 9 & 5 & 5 & 19 & 8.3 \\
\hline \multicolumn{6}{|l|}{ Marital status } \\
\hline Single & 3 & 4 & 3 & 10 & 4.3 \\
\hline Married monogamous & 59 & 49 & 39 & 147 & 63.6 \\
\hline Married polygamous & 28 & 25 & 17 & 70 & 30.3 \\
\hline Divorce & 1 & 0 & 0 & 1 & 0.4 \\
\hline Co-habiting & 0 & 0 & 1 & 1 & 0.4 \\
\hline Widow & 0 & 2 & 0 & 2 & 0.9 \\
\hline
\end{tabular}

JTH - Juba Teaching Hospital

MNHIC $=$ Malaki National Health Insurance Centre

MPHCC $=$ Munuki Primary Health Care Centre

Of the 231 pregnant women participants, 51 (22.1\%) were positive for rapid plasma reagin (RPR) test 147 and $79(34.2 \%)$ were positive for Treponema pallidum haemagglutination assay. There was no significant difference between prevalence of syphilis in the three study sites. Using the RPR test, the detection ranged from a low of $0.9 \%$ in the $35-39$ years age group to high of $8.7 \%$ in the $25-29$ years age group. Pregnant women with a gestation period of 24-30 weeks were most affected giving a prevalence rate of $6.9 \%$. Educational levels of the participants with positive tests for syphilis as a proportion of all the study participants ranged from either not having or having only primary level education $(15.6 \%)$ to having tertiary education $(2.2 \%)$.

More than three quarters of the $22.1 \%$ who were positive for syphilis were housewives giving a prevalence of $16.5 \%$ while those who were in informal employment had the lowest prevalence of $0.4 \%$. In this study syphilis was not detected in either single or divorced pregnant women participants while it was detected in $14.7 \%$ of the married monogamous and in $6.5 \%$ in the married polygamous (Table 2). 
Table 2

Prevalence of syphilis by socio-demographic status of pregnant women

\begin{tabular}{|c|c|c|c|c|c|}
\hline Variable & Total (n) & $\begin{array}{l}\text { RPR } \\
\text { Positive } \\
\text { No. }(\%)\end{array}$ & $\begin{array}{l}\text { RPR } \\
95 \% \text { CI }\end{array}$ & $\begin{array}{l}\text { TPHA } \\
\text { Positive } \\
\text { No. }(\%)\end{array}$ & $\begin{array}{l}\text { TPHA } \\
95 \% \text { CI }\end{array}$ \\
\hline \multicolumn{6}{|l|}{ Age group } \\
\hline 15-19 & 44 & $8 \quad 3.5$ & $9-35$ & $9 \quad 3.9$ & $5.3-20.5$ \\
\hline $20-24$ & 87 & 166.9 & $11-28$ & 2912.6 & $26-48.3$ \\
\hline $25-29$ & 70 & 208.7 & $19-42$ & 3013.0 & $27.3-49.6$ \\
\hline $30-34$ & 25 & $5 \quad 2.1$ & $9-41$ & $7 \quad 3.03$ & $3.6-17.4$ \\
\hline $35-39$ & 7 & 20.9 & $3.7-71$ & 1.7 & $1.4-12.5$ \\
\hline \multicolumn{6}{|l|}{ Gestation period in weeks } \\
\hline $6-12$ & 26 & 73 & $11.1-45$ & 93.9 & $4.5-25.9$ \\
\hline $12-18$ & 22 & $4 \quad 1.7$ & $5.2-40.3$ & $8 \quad 3.5$ & $4.5-19.0$ \\
\hline $18-24$ & 65 & 166.9 & $15.8-37$ & 229.5 & 18.3-19.1 \\
\hline $24-30$ & 52 & 135.6 & $14-39$ & 177.4 & $13.1-32.2$ \\
\hline $30-36$ & 65 & 114.8 & $8.8-28.3$ & 2310.0 & $19.4-4.6$ \\
\hline$>36$ & 1 & $0 \quad 0$ & $0-97.5$ & $0 \quad 0$ & $0.0-4.5$ \\
\hline \multicolumn{6}{|l|}{ Education } \\
\hline None & 71 & 229.5 & $20.5-43.1$ & 3615.6 & $34.3-57.2$ \\
\hline Primary & 80 & 146.1 & $10-27.6$ & 2510.8 & $21.6-43.1$ \\
\hline Secondary & 57 & 104.3 & $8.7-30$ & 114.8 & $7.2-23.5$ \\
\hline Tertiary & 23 & 52.2 & $7.5-43.7$ & $7 \quad 3.03$ & 3.6-17.4 \\
\hline \multicolumn{6}{|l|}{ Occupation } \\
\hline Housewife & 176 & 3816.5 & $15.8-28$ & 6628.6 & $73.5-90.9$ \\
\hline Employed & 36 & 125.2 & $19.1-52.2$ & 114.8 & $7.2-23.5$ \\
\hline Informal employment & 19 & 10.4 & $0.1-26$ & 0.9 & $0.3-8.8$ \\
\hline \multicolumn{6}{|c|}{ Marital status } \\
\hline Single & 10 & $0 \quad 0$ & $0.0-30.8$ & 0.8 & $23.3-10.3$ \\
\hline Monogamous & 147 & 3414.7 & $16.6-30.8$ & $47 \quad 2003$ & $57.7-73.3$ \\
\hline Polygamous & 70 & 156.5 & $12.5-32.9$ & 2711.6 & $21.3-36.2$ \\
\hline Divorced & 1 & $0 \quad 0$ & $0.0-97.5$ & 10.4 & $0.0-2.5$ \\
\hline Co-habiting & 2 & 10.43 & $1.3-98.7$ & 0.4 & $0.0-3.6$ \\
\hline Widow & 1 & 10.43 & $100-100$ & 0.4 & $0.0-2.4$ \\
\hline Total & 231 & $\begin{array}{ll}51 & 22.1\end{array}$ & & 7934.2 & \\
\hline
\end{tabular}

Unconditional logistic regression on factors associated with syphilis revealed that the risk factors significantly associated with syphilis were: being a housewife (OR 2.808; $p=0.0116$ ), having a history of abortion
$(\mathrm{OR} 2.654 ;=0.0116)$ and a partner's history of regular travel(OR 2.149; $\mathrm{p}=0.028)$. Attending antenatal clinic for previous pregnancy was significantly protective (OR 0.281; $\mathrm{p}=0.0004)$ (Table 3). 
Table 3

Unconditional logistic regression final model for factors associated with syphilis in pregnant women

\begin{tabular}{lcccc} 
Factor & Odds Ratio & $95 \%$ & CI & P-value \\
\hline Housewife (Yes/No) & 2.8082 & 1.2592 & 6.2625 & 0.0116 \\
History of abortion/ child death (Yes/No) & 2.6548 & 1.2442 & 5.6648 & 0.0116 \\
Attending ANC for previous pregnancy (Yes/No) & 0.2814 & 0.1403 & 0.5643 & 0.0004 \\
History of partner travelling (Yes/No) & 2.1491 & 1.0833 & 4.2633 & 0.0286 \\
\hline
\end{tabular}

ANC $=$ Antenatal Clinic

\section{DISCUSSION}

Syphilis prevalence was normally distributed between all the age groups in this study; that is the lower age group of 15 to 19 years and the higher age group of 35 to 39 years had lower prevalence as opposed to the age group of 25 to 29 years which had the highest prevalence hence majority of the study participants also fell in this age group. This finding is in agreement with studies done in Northwest Ethiopia which also found the high prevalence to be among the same age group (6), and contrasts with the findings from South Africa, which reported high prevalence of maternal syphilis to be among age group of 35 to 39 years (7).

The overall prevalence of syphilis among pregnant women in selected antenatal clinics in Juba from the RPR results was $22.1 \%$. The prevalence of syphilis was high in pregnant women with a gestation period of 18-24 weeks which is the second trimester of pregnancy. This finding could be attributed to a possible common behaviour or practice where pregnant women tend to utilise antenatal care clinics from their second trimester of pregnancy. However, a study from Ethiopia found that majority of pregnant women with syphilis were in their third trimester of pregnancy (8). There is no significant difference with the findings from Southern Sudan sentinel surveillance report of 2006 which found a prevalence which ranged between $12 \%$ to $21 \%$. In Kinshasa, Zaire the prevalence was found to be $16 \%$ (9) and $13.7 \%$ in North-west Ethiopia (8).

Recently a similar study was also done in Khartoum, the capital of Sudan in which maternal syphilis of $9 \%$ among the antenatal care attendees was reported (10), thisis lower than the current study. These differences could be due to the better health system in Khartoum as opposed to that in Juba which is yet to be established to support maternal and child health. The prevalence results from this current study are different from those found by other workers who found very low rates ranging from a low of $0 \%$ in Kabul, Afghanistan (11), $0.9 \%$ in a suburban community in Northern
Sudan (12) and $1.03 \%$ in South Africa (13). Other low prevalence of $4.0 \%$ in pregnant women has been found in Entebbe, Uganda (14). These variations from different countries or sites might be due to studies done after interventional programmes which might have reduced the prevalence of maternal syphilis in those areas. The high prevalence of syphilis in Juba observed by this study could be due to lack of intervention programmes set in place to reduce the prevalence which may also lead to reduction of maternal negative outcomes associated with syphilis.

Several potential factors associated with syphilis were measured in this study for statistical association. The initial univariate analysis indicated that having low education, being a housewife, history of abortion or early child death and history of partner travel were risk factors for having syphilis; meanwhile attending antenatal clinic for previous pregnancy was found to be a protective factor ( $p$ $<0.05$ ). These findings are in agreement with several studies carried out in Nairobi (15) and in China (16). However these same factors and others with $p$-value $\leq 0.1$ were subjected to multivariate analysis; and being a housewife, history of abortion or child death and history of partner travel were risk factors while attending antenatal clinic for previous pregnancy remained a protective factor. This is in agreement with study in Shenzhen, China (16). The study participants who reported history of frequent travel of their partner were more likely to be positive for syphilis than those with no history of travel by their partner. There is a high tendency for the partners to travel to other towns and states in Sudan as they are more often than not the sole breadwinners for their families and have to seek employment out of town. This observation has also been reported in China (16).

Being a housewife was associated with syphilis in pregnant women in this study, where being a housewife is associated with lack of education and employment and this contrasts with the study done in China which found that having informal job was a risk factor for syphilis (17). 
Attending antenatal care clinic for previous pregnancy was found to be a protective factor for syphilis in pregnant women. This is an important finding that could explain the fact that pregnant women who utilise the antenatal clinic have clear knowledge of syphilis or maternal and child health; likewise a study in Kwale district in Kenya showed the same finding with this study (18). Marital status was not associated with syphilis in this study although majority of the study participants were in monogamous marriages, unlike another study which found significant association between being single or in a polygamous marriage as risk factors for syphilis in pregnant women (19). Other factors were knowledge of syphilis, and previous history of giving birth which however were not statistically significant with having syphilis in this study.

Rapid plasma reagin test has been used as a syphilis screening test in many antenatal clinics and health facilities in the developing world (20). To define active syphilis the RPR and the TPHA positive tests were compared. The difference between the high positivity in TPHA could be due to past exposure to syphilis. The TPHA test is capable of detecting past infection by detecting both immunoglobulins IgG and IgM. TPHA testing was used in this current study as a confirmatory test with very good results.

There is a very urgent need for strategic interventional programme to improve pre-natal and neonatal outcomes particularly in Juba and generally in South Sudan. This will have the effect of reducing the prevalence of syphilis among pregnant women. Many African and other developing countries have adopted screening and treatment of pregnant women for syphilis in the first antenatal visit, which have resulted in reduction of syphilis and its negative outcome in pregnancy and later life. Regular health education for pregnant women in antenatal clinics, to inform them about their health and the risk of syphilis to both born and the un-born child are required.

\section{ACKNOWLEDGEMENTS}

To the Ministry of Health of the Government of Southern Sudan for granting us the permission to conduct this study. We acknowledge M. Yuasa for assisting in data collection and storage. We also thank A. Poni for assisting in the processing and analysis of specimen in the laboratory. This work was part of a Master of Science in Laboratory Management and Epidemiology for S.K. Emmanuel at the Institute of Tropical Medicine and Infectious Diseases of the Jomo Kenyatta University of Agriculture and Technology.

\section{REFERENCES}

1. Walker, G.J.A. Antibiotics for syphilis diagnosed during pregnancy (Protocol for a Cochrane Review). Issue 3: Oxford:UpdateSoftware. Level IV The Cochrane Library J. Clin. Path.

2. Hurtig, A-K., A., Nicoll, A., Carne, C., et al. Syphilis in pregnant women and their children in the United Kingdom: Res. Nat. Clin. Rep. Surv. 1998; 317: 16171619.

3. Watson-Jones, D., Changalucha, J., Gumodoka B., et al. Syphilis in pregnancy in Tanzania. Impact of maternal syphilis on outcome of pregnancy. J. Infect. Dis. 2002; 186: 940-947.

4. Gerbase, A.C., Rowley, J.T., and Mertens, T.E. Global epidemiology of sexually transmitted diseases. Lancet. 1998; 351 (suppl III): SIII 2-4.

5. Folgosa, E., Bique, O., Gonzalezt, C., et al. Syphilis seroprevalence among pregnant women and its role as a risk factor for stillbirth in Maputo, Mozambique. Genitourin. Med. 1996; 72: 339-342.

6. Mulu, A., Kasu, A., Tessema, B., V. et al.Seroprevalence of syphilis and HIV-l during pregnancy in a teaching hospital in North west of Ethiopia. J. Inf. Dis. 2007; 60: 193-195.

7. Swai, R., Geofrey, R. S., Mecky M., et al. Surveillance of HIV and syphilis infections among antenatal clinic attendees in Tanzania. BMC Pub. Hlth. 2006; 6:91 doi: 10.1186/1471-2458-6-91.

8. Azeze, B., Fantahun, M., Kidan, K. and Haile, T. Seroprevalence of syphilis amongst pregnant women attending antenatal clinics in a rural hospital in North West Ethiopia. BMC Inf. Dis.1995; 8: 119 doi: 10.1186/1471-2334-8-119.

9. Goeman, J., Kivuvu, M., Nzila, N., et al. Similar serological response to conventional therapy for syphilis among HIV-positive and HIV-negative women. Genitourin. Med. 1995 71: 275-279.

10. Abdelbagi, M. N., Hager, A. W., and Omer, M. K. Seroprevalence of syphilis among pregnant women in the Tri-capital, Khartoum, Sudan. J. Med. Sci. 2008; 1: 48-52.

11. Todd,C.,Ahmadzai,M.,Atiqzai,F., etal.Seroprevalence and correlates of HIV, syphilis, and hepatitis B and $C$ virus among intrapartum patients in Kabul, Afghanistan. BMC Infect. Dis. 2008; 8: 1-9.

12. Kafi, S., Abdelrahim, M. and Hassan, M. Prevalence of sexual transmitted disease among women in suburban communities in Northern Sudan. Upsala J. Med. Sci. 2000; 105: 249-254. 
13. Mullick, S., Beksinksa M. and Msomi, S. Treatment for syphilis in antenatal care: compliance with the three dose standard treatment regimen. Sex Transm. Infect. 2005; 81: 220-222.

14. Kizito, D., Patrick, W., Kesande, B., et al. Uptake of HIV and syphilis testing of pregnant women and their male partners in a programme for prevention of mother-to-child HIV transmission in Uganda. Trop. Med. Intern. Hlth. 2008; 135: 680-682.

15. Temmerman, M., Gichangi P., Fonck K., et al. Effect of a syphilis control programme on pregnancy outcome in Nairobi, Kenya. J. Sex. Transm. Infect. 2000; 76: 117121.

16. Zhou, H., Chen, X., Fu-C., et al. Risk factors for syphilis infection among pregnant women: results of a casecontrol study in Shenzhen, China. J. Sex Transm. Infect. 2007; 83: 476-480

17. Cheng, J., Zhou, H., Hong, F., et al. Syphilis screening and intervention in 500000 pregnant women in
Shenzhen, the People's Republic of China. J. Sex Transm. Inf. 2007; 83: 347-350.

18. Brown, C., Sohani S., Khan K., Lilford, R. and Mukhwana, W. Antenatal care and perinatal outcomes inKwale district, Kenya. Pregnancy and childbirth. BMC Preg. Childbirth. 2008; 8: 2 doi:10.1186/1471-2393-8-2.

19. Swai, R., Geofrey, R. S., Mecky, M., et al. Surveillance of HIV and syphilis infections among antenatal clinic attendees in Tanzania. BMC Pub. Hlth. 2006; 6: 91 doi: $10.1186 / 1471 ; .2458-6-91$.

20. West, B., Walgreen, G., Morison, L., Brouwers, J. and Bailey R. Performance of the rapid plasma reagin and the rapid syphilis screening tests in the diagnosis of syphilis in field conditions in rural Africa. Sex Transm. Infect. 2002; 78: 282-285. 